\title{
Phospholipase $C \varepsilon$ plays a crucial role in neutrophilic inflammation accompanying acute lung injury through augmentation of CXC chemokine production from alveolar epithelial cells
}

Kanoko Umezawa ${ }^{1}$, Tatsuya Nagano ${ }^{1 *} \mathbb{D}$, Kazuyuki Kobayashi', Ryota Dokuni ${ }^{1}$, Masahiro Katsurada', Masatsugu Yamamoto', Yoko Yoshikawa², Tohru Kataoka ${ }^{2,3}$ and Yoshihiro Nishimura'

\begin{abstract}
Background: We have shown that phospholipase $C \varepsilon(P L C \varepsilon)$, an effector of Ras and Rap1 small GTPases, plays pivotal roles in inflammation and inflammation-associated carcinogenesis by augmenting proinflammatory cytokine production from epithelial cells of various organs. The purpose of this study is to analyze its role in neutrophilic alveolar inflammation accompanying acute lung injury (ALI), focusing on that in alveolar epithelial cells (AECs), which are known to make a major contribution to the pathogenesis of ALI.

Methods: We examine the effect of the PLCE genotypes on the development of ALI induced by intratracheal administration of lipopolysaccharide (LPS) to $P L C \varepsilon$ wild-type $\left(P L C \varepsilon^{+/+}\right)$and knockout $\left(P L C \varepsilon^{\Delta X \Delta X}\right)$ mice. Pathogenesis of ALI is analyzed by histological examination of lung inflammation and measurements of the levels of various cytokines, in particular neutrophil-attracting chemokines such as $\mathrm{CxCl5}$, by quantitative reverse transcriptionpolymerase chain reaction and immunostaining. Primary cultures of AECs, established from $P L C \varepsilon^{+/+}$and $P L C \varepsilon^{\Delta x \Delta x}$ mice, are used to analyze the roles of PLC $\varepsilon$, protein kinase D (PKD) and nuclear factor-KB (NF-KB) in augmentation of LPS-induced Cxcl5 expression.

Results: Compared to $P L C \varepsilon^{+/+}$mice, $P L C \varepsilon^{\Delta x \Delta x}$ mice exhibit marked alleviation of lung inflammation as shown by great reduction in lung wet/dry weight ratios, accumulation of inflammatory cells in the alveolar space and thickening of alveolar walls as well as the number of neutrophils and the protein concentration in bronchoalveolar lavage fluid. Also, LPS-induced expression of the CXC family of chemokines, in particular CXCl5, is substantially diminished in the total lung and AECs of $P L C \varepsilon^{\Delta X / \Delta X}$ mice. Moreover, LPS-induced CxCl5 expression in primary cultured AECs is markedly suppressed on the $P L C \varepsilon^{\Delta X / \Delta X}$ background $\left(p<0.05\right.$ versus $P L C \varepsilon^{+++}$AECs), which is accompanied by the reduction in phosphorylation of inhibitor KB (IKB), PKD and nuclear translocation of NF-KB p65. Also, it is suppressed by the treatment with inhibitors of PKD and IKB kinase, suggesting the involvement of the PLCE-PKD-IKB-NF-KB pathway.

(Continued on next page)
\end{abstract}

\footnotetext{
* Correspondence: tnagano@med.kobe-u.ac.jp

${ }^{1}$ Division of Respiratory Medicine, Department of Internal Medicine, Kobe University Graduate School of Medicine, 7-5-1 Kusunoki-cho, Chuo-ku, Kobe 650-0017, Japan

Full list of author information is available at the end of the article
}

(c) The Author(s). 2019 Open Access This article is distributed under the terms of the Creative Commons Attribution 4.0 International License (http://creativecommons.org/licenses/by/4.0/), which permits unrestricted use, distribution, and reproduction in any medium, provided you give appropriate credit to the original author(s) and the source, provide a link to the Creative Commons license, and indicate if changes were made. The Creative Commons Public Domain Dedication waiver (http://creativecommons.org/publicdomain/zero/1.0/) applies to the data made available in this article, unless otherwise stated. 
(Continued from previous page)

Conclusions: PLCE-mediated augmentation of the production of the CXC family of chemokines, in particular CxCl5, in AECs plays a crucial role in neutrophilic alveolar inflammation accompanying ALI, suggesting that PLC $\varepsilon$ may be a potential molecular target for the treatment of acute respiratory distress syndrome.

Keywords: Phospholipase $C \varepsilon$, Acute lung injury, Acute respiratory distress syndrome, Alveolar epithelial cells, Inflammation, Mouse model, Neutrophil, Nuclear factor-kB, Cxcl5,

\section{Background}

Acute respiratory distress syndrome (ARDS) is a life threatening respiratory condition characterized by tachypnea, refractory hypoxemia and diffuse opacities on chest radiographs following sepsis, pneumonia and trauma [1]. The mortality of ARDS patients is around 35-40\%, however, effective protocols for its treatment remains to be worked out despite exhaustive investigation [2]. The pathological development of ARDS is traditionally classified into 3 successive phases: exudative, proliferative and fibrotic [1]. The exudative phase is characterized by immune-cell-mediated damage to alveolar epithelial cells (AECs) and endothelial cells and by the dysfunction of the alveolar capillaries. In the proliferative phase, the numbers of abnormal type II alveolar cells and inflammatory cells are increased, and, in the fibrotic phase, fibroblasts infiltrate and replace cells in the alveoli and alveolar ducts as fibrosis develops. The current view of ARDS suggests that these 3 phases are overlapping with each other [3] . Classically, the most characteristic feature of ARDS is inflammation with heavy infiltration of neutrophils, which is mediated by elevation of proinflammatory cytokines such as interleukin (IL)-1 $\beta$, IL-6, IL-8 and tumor necrosis factor- $\alpha$ (TNF- $\alpha$ ). Additionally, leukocyte recruitment is orchestrated bychemokines, in particular the neutrophil-attracting chemokines, $\mathrm{C}-\mathrm{X}-\mathrm{C}$ motif ligands (CXCLs) and $\mathrm{C}-\mathrm{C}$ motif ligands (CCLs) $[1,4]$. The injured AECs act as the main source of these chemokines, thereby making a great contribution to the pathogenesis of ARDS [5]. In this study, we focuse on the role of $\mathrm{Cxcl} 5$, which is produced from AECs, different from myeloid cells which produce most chemokines and reported to play a key role in neutrophil activation during the pathogenesis of ARDS [6].

Phospholipase C (PLC) hydrolyzes phosphatidylinositol 4,5-bisphosphate to generate inositol 1,4,5-triphosphate and diacylglycerol (DAG), which function as vital intracellular second messengers through mobilization of $\mathrm{Ca}^{2+}$ from the intracellular stores and activation of DAG-target proteins such as protein kinases $C$ and $D$ (PKD), respectively [7]. Thirteen PLC isoforms are present in mammals and grouped into 6 classes: $\beta, \gamma, \delta, \varepsilon, \zeta$, and $\eta$, based on the similarities in their structures and regulatory mechanisms. Among them, PLCe is the only isoform regulated by small GTPases Ras and Rap [8], and further studies showed that it is also regulated by Rho small GTPases and heterotrimeric $G$ protein $G \alpha_{12}, G \alpha_{13}$ and $\beta_{1} \gamma_{2}$ subunits [7]. Stimulation of $G$ protein-coupled receptors (GPCRs) with their ligands such as lysophosphatidic acid (LPA), sphingosine-1-phosphate (S1P) and thrombin induces PLC $\varepsilon$ activation [9]. PLCe is expressed in structural cells such as epithelial cells and endothelial cells but not in immune cells such as granulocytes, lymphocytes and macrophages [10-12]. By utilizing genetically modified mice for $P L C \varepsilon$, we demonstrated that PLCE plays a crucial role in experimentally induced inflammation; mice homozygous for the inactivated PLCEalleles $\left(P L C \varepsilon^{\Delta X / \Delta X}\right.$ mice) exhibited markedly attenuated inflammatory responses in various animal models including the phorbor ester-induced dermatitis, hapten-induced contact dermatitis and dextran sulfate-induced colitis models $[10,11,13]$ and transgenic mice overexpressing PLCE specifically in the skin keratinocytes spontaneously developed chronic dermatitis resembling human psoriasis [14]. Concurrently, $P L C \varepsilon^{\Delta X / \Delta X}$ mice showed marked resistance to tumor formation in the two-stage skin chemical carcinogenesis and the de novo intestinal carcinogenesis on the $A P C^{\mathrm{Min} /+}$ background, which were associated with attenuation of cancer-associated inflammation $[15,16]$. In the lung, we found that PLCE plays a crucial role in Th2-cell-mediated eosinophilic inflammation in the mouse model of ovalbumin-sensitized allergic bronchial asthma [12]. In this model, primary culture of bronchial epithelial cells was used to demonstrate that PLCE is required for TNF- $\alpha$-induced pro-inflammatory cytokine production. Recently, we showed that, in human colon epithelial Caco2 cells, PLC $\varepsilon$ enhances pro-inflammatory cytokine expression through nuclear factor- $\mathrm{kB} \quad(\mathrm{NF}-\mathrm{\kappa} \mathrm{B})$ activation through activation of $\mathrm{PKD}$, thereby recruiting and activating immune cells to initiate and sustain inflammation [13]. It was shown that the PLCE-PKD axis, activated by LPA receptor engagement, augments cytokine production via NF- $\mathrm{KB}$ activation through phosphorylation and degradation of inhibitor $\kappa \mathrm{B}$ (IкB) by ribosomal S6 kinase (RSK), not by IкB kinase (IKK) in the canonical NF- $\mathrm{kB}$ pathway.

Thus, we examine the role of PLCE in AECs in the pathogenesis of experimentally induced ARDS by employing the mouse model of LPS-induced ALI. 


\section{Methods}

Reagents

LPS from Escherichia coli O111:B4 (L2630, Sigma-Aldrich, St. Louis, MO, USA), IKK inhibitor BMS-345541 (401,480, Calbiochem, Darmstadt, Germany), PKD inhibitor CID755676 (476,495, Calbiochem) and proteasome inhibitor MG-132(Calbiochem) were commercially obtained. Primary antibodies against the following proteins were purchased from Cell Signaling Technology (Denver, MA, USA): $\beta$-actin (\#4967), phospho-IкB $\alpha$ (Ser32) (14D4) (\#2859), ІкB $\alpha$ (\#9242), phospho-PKD (Ser916) (\#2051) and PKD (\#2052). Other antibodies used were anti-CD45 (30-F11, eBioscience, San Diego, CA, USA), anti-CD32 (93, eBioscience), anti-Cxcl5 (PAA860Mu01, Cloud-Clone Corp, Katy, TX), anti-pan-cytokeratin (C-11, ab7753, Abcam, Cambridge, UK), anti-prosurfactant protein $\mathrm{C}$ (pro-SPC) (ab90716, Abcam), anti-podoplanin (bs-1048R, Bioss, Boston, MA, USA) and anti-NF-kB p65 (sc-109, Santa Cruz, Dallas, TX, USA). In addition, CF dye-labeled secondary antibodies, CF488A (20015) and CF555 (20231), were purchased from Biotium (Hayward, CA, USA).

\section{Animals}

$P L C \varepsilon^{\Delta X / \Delta X}$ mice homozygous for the allele harboring an in-frame deletion of the catalytic X domain of PLC $\varepsilon$ and hence devoid of its lipase activity [15] were backcrossed to C57BL6JJcl mice (CLEA Japan, Tokyo, Japan) for at least 8 generations. All animals were maintained in the animal facility of Kobe University Graduate School of Medicine. The use and care of animals were reviewed and approved by the Institutional Animal Care and Use Committee of Kobe University (Permit Numbers: P130612, P130612-R1 and P130401).

\section{AEC culture}

Primary culture of type II AECs was prepared from adult naïve $P L C \varepsilon^{+/+}$and $P L C \varepsilon^{\Delta X / \Delta X}$ mice according to the previously described protocol [17-19]. Culture dishes $(\Phi=$ $10 \mathrm{~cm})$ coated with anti-mouse CD45 antibody $(40 \mu \mathrm{g})$ and anti-mouse CD32 antibody $(15 \mu \mathrm{g})$ were used to get rid of leukocytes from the culture. The epithelial nature of the cultured cells was confirmed by positive pan-cytokeratin staining in at least $97 \%$ of the cells. They were further checked by positive staining for pro-SPC and podoplanin, specific markers of AECs (Additional file 1: Figure S1). On day 3, the cells were stimulated with LPS $(500 \mathrm{ng} / \mathrm{mL})$ for various times $[6,20]$ and used for further analyses. When necessary, the cells were treated with the IKK inhibitor $(20 \mu \mathrm{M})$ for $30 \mathrm{~min}$ or with the PKD inhibitor $(10 \mu \mathrm{g} / \mathrm{ml})$ for $60 \mathrm{~min}$ before LPS stimulation.

\section{Quantitative reverse transcription-polymerase chain reaction (qRT-PCR)}

Preparation of total cellular RNAs and qRT-PCR using them as a template were performed as described previously [12]. The primers used for the qRT-PCR analyses of various chemokine mRNAs are summarized in Additional file 2: Table S1. The relative mRNA levels were calculated according to the $\Delta \Delta \mathrm{Ct}$ method using the $\beta$-actin mRNA as an internal control.

\section{Western immunoblotting and immunofluorescence staining analyses for cytokines expressed in AECs} Cultured AECs were lysed in Cell Lysis Buffer (Cell Signaling Technology) and total cellular proteins $(20 \mu \mathrm{g})$ were separated by sodium dodecyl sulfate-polyacrylamide gel electrophoresis (SDS-PAGE), followed by Western immunoblotting as described before [21]. Immunofluorescence staining was performed as previously described with minor modifications [22].

\section{Induction of $A L I$ by LPS administration}

$P L C \varepsilon^{+/+}$and $P L C \varepsilon^{\Delta \mathrm{X} / \Delta \mathrm{X}}$ mice at $8-10$ weeks of age were anesthetized by intraperitoneal injection of dexmedetomidine ( $40 \mu \mathrm{l}$; Maruishi Pharmaceutical, Osaka, Japan), midazolam (11 $\mu \mathrm{l}$; Astellas Pharma, Tokyo, Japan) and butorphanol tartrate $(13 \mu \mathrm{l}$; Meiji Seika Pharma, Tokyo, Japan). The mice were randomly divided into the ALI model group and the control group, and received intratracheal administration of $50 \mu \mathrm{l}$ phosphate-buffered saline (PBS) with and without LPS $(5 \mathrm{mg} / \mathrm{kg})$, respectively [23]. Twenty-four hours later, the mice were sacrificed for dissection of the lungs or subjected to the collection of bronchoalveolar lavage fluid (BALF).

\section{Collection and analysis of BALF}

BALF samples were collected by washing the lung three times with $0.8 \mathrm{ml}$ PBS through a tracheal cannula placed into each mouse under anesthesia as described previously [12]. The number of leukocytes in the BALF was counted using a hemocytometer. The BALF was then centrifuged at $1500 \times \mathrm{rpm}$ for $10 \mathrm{~min}$ at $4{ }^{\circ} \mathrm{C}$, and the cell pellet was suspended in $50 \mu \mathrm{l} \mathrm{PBS}$, subjected to cytospin preparation and stained by Romanowski protocol using Diff-Quik (Sysmex, Kobe, Japan). At least 200 leukocytes on each slide were subjected to differential counting of macrophages, neutrophils, lymphocytes and eosinophils according to the standard morphological criteria.

\section{Protein and cytokine measurements in BALF}

The concentration of total protein in BALF was measured by using the Bradford protein assay (Bio-Rad, Hercules, CA, USA). The concentration of $\mathrm{Cxcl} 5$ in BALF was determined using the ELISA kit (R\&D systems, Minneapolis, MN, USA). 


\section{Histopathologic and imunohistochemical analyses}

The dissected lungs were filled with phosphate-buffered paraformaldehyde via intratracheal instillation and further fixed in phosphate-buffered paraformaldehyde. The lung tissues were embedded in paraffin, sectioned and subjected to staining with hematoxylin and eosin $(\mathrm{H} \& \mathrm{E})$. The paraffin-embedded sections were also subjected to immunohistochemical staining with the anti-Cxcl5 antibody $(15 \mu \mathrm{g} / \mathrm{ml})$ and the anti-pro-SPC antibody $(1 \mu \mathrm{g} /$ $\mathrm{ml}$ ) along with the peroxidase polymer anti-rabbit IgG reagents by using the Polymer method with ImmPRESS reagent (Vector Laboratories, Burlingame, CA, USA).

\section{Measurements of the lung wet/dry weight ratio}

The dissected lung was immediately weighed to measure the wet weight. Subsequently, it was dried in an incubator at $60^{\circ} \mathrm{C}$ for $24 \mathrm{~h}$ until its weight was stabilized and was subjected to the measurement of the dry weight. The wet/dry weight ratio was calculated by dividing the wet weight by the dry weight.

\section{Statistical analyses}

Data are presented as the mean \pm standard deviation (SD). Statistical analyses were performed using the Student's unpaired $t$-test to compare the differences between two groups. If a $p$-value was smaller than 0.05 , the difference was considered statistically significant.

\section{Results}

\section{Alleviation of $A L I$ in $P L C \varepsilon^{\Delta X / \Delta X}$ mice}

To analyze the effect of the PLCE genetic background, the experimental model of LPS-induced ALI was applied to $P L C \varepsilon^{+/+}$and $P L C \varepsilon^{\Delta X / \Delta X}$ mice. Twenty-four hours after the LPS administration, we examined the features a

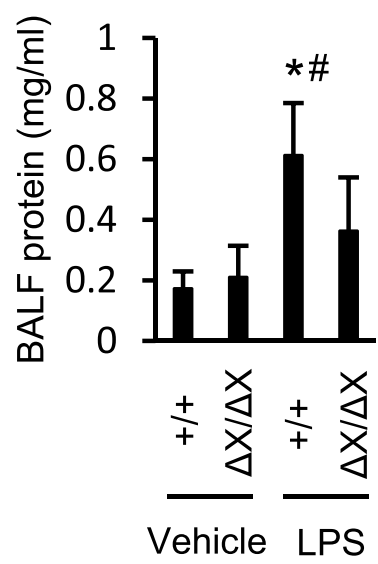

b

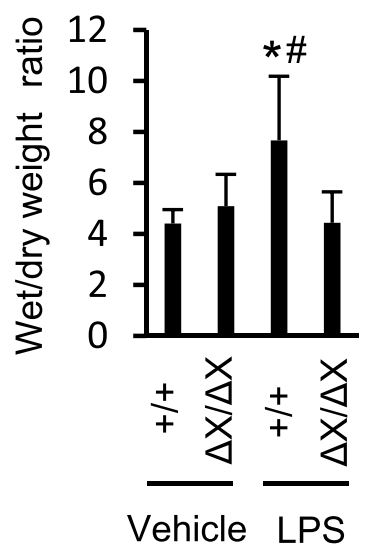

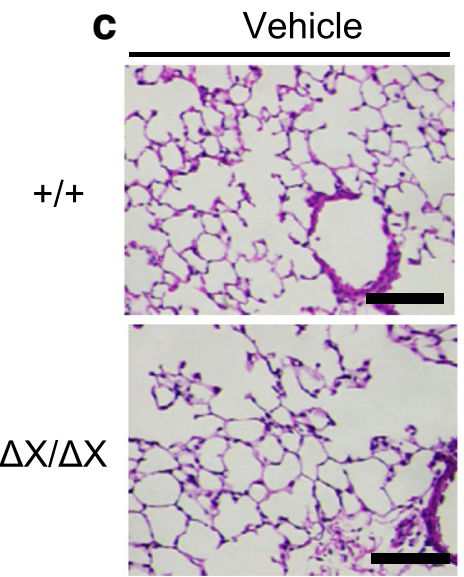

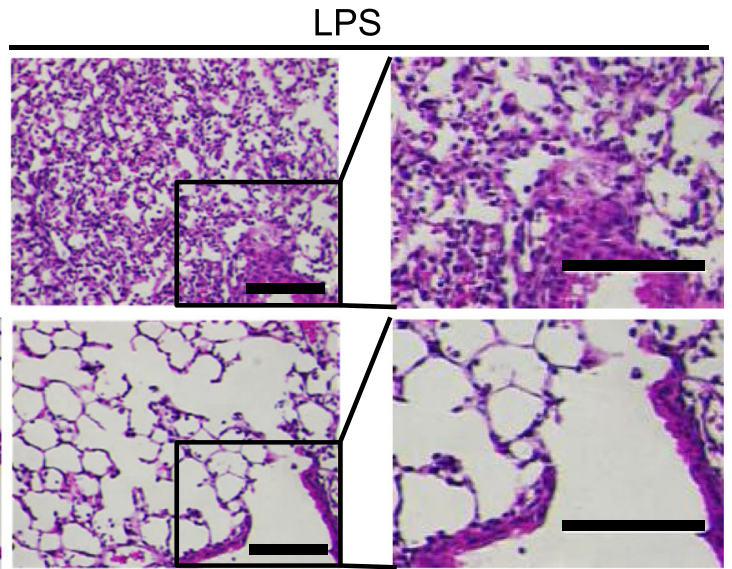

Fig. 1 Effects of the PLCE genotypes on the development of LPS-induced ALI. a Effects on LPS-induced alteration of the alveolar capillary barrier . The total protein in BALF was measured. BALF was collected in $24 \mathrm{~h}$ after i.t. administration of LPS $(5 \mathrm{mg} / \mathrm{kg})$ or vehicle. $n=9$ in the LPS-treated group, and $n=6$ in the vehicle-treated group. ${ }^{*} p<0.05$ between control and LPS administration, \#, $p<0.05$ between PLCE genotypes. b Lung edema was evaluated by determining the wet/dry ratios of the lung tissue isolated from mice in $24 \mathrm{~h}$ after i.t. administration of LPS or vehicle ( $n$ $=8$ in the LPS-treated group and $n=4$ in the vehicle-treated group). ${ }^{*}, p<0.05$ between control and LPS administration, \#, $p<0.05$ between PLC $\varepsilon$ genotypes. c Histologic analysis of the lung. Lung sections were prepared in $24 \mathrm{~h}$ after administration of LPS or vehicle as in $\mathbf{a}$, and they were stained with H\&E. $n=3$ in each group. Scale bar, $100 \mu \mathrm{m}$ 
characteristic of the experimental ALI. The increase in the protein concentration in BALF following the LPS administration, observed in $P L C \varepsilon^{+/+}$mice, was attenuated in $P L C \varepsilon^{\Delta X / \Delta X}$ mice $(p<0.05$, Fig. 1a). Concurrently, the LPS-induced increase in the lung wet/dry weight ratios, observed in $P L C \varepsilon^{+/+}$mice, was greatly attenuated in $P L C \varepsilon^{\Delta X / \Delta X}$ mice $(p<0.05$, Fig. $1 \mathrm{~b})$. These results indicated that both the increase in pulmonary permeability and the development of pulmonary edema were alleviated on the $P L C \varepsilon^{\Delta X / \Delta X}$ background. Histological analysis of the lung tissues revealed that $P L C \varepsilon^{\Delta X / \Delta X}$ mice exhibited great reduction in the LPS-induced accumulation of inflammatory cells in the alveolar space, which was indicative of attenuation of lung inflammation (Fig. 1c). Moreover, the LPS-induced thickening of alveolar walls, observed in $P L C \varepsilon^{+/+}$mice, was substantially suppressed in $P L C \varepsilon^{\Delta X / \Delta X}$ mice, which was indicative of attenuation of the development of the alveolar capillary dysfunction.

As neutrophils are known to play a crucial role in ALI, we examined the nature of the leukocytes infiltrated into the lungs by analyzing BALF collected in $24 \mathrm{~h}$ after the LPS administration. Differential leukocyte counting revealed that the LPS-induced increase in the total number of leukocytes in the BALF, observed in $P L C \varepsilon^{+/+}$ mice, was greatly suppressed in $P L C \varepsilon^{\Delta X / \Delta X}$ mice $(p<$ 0.05 , Fig. 2) and that the leukocytes were predominantly composed of neutrophils, whose number was also diminished in $P L C \varepsilon^{\Delta X / \Delta X}$ mice $(p<0.05$, Fig. 2). The number of macrophages failed to show an increase depending on the LPS stimulation or the $P L C \varepsilon^{+/+}$ background (Fig. 2a). These results demonstrated that PLCE plays a crucial role in neutrophilic
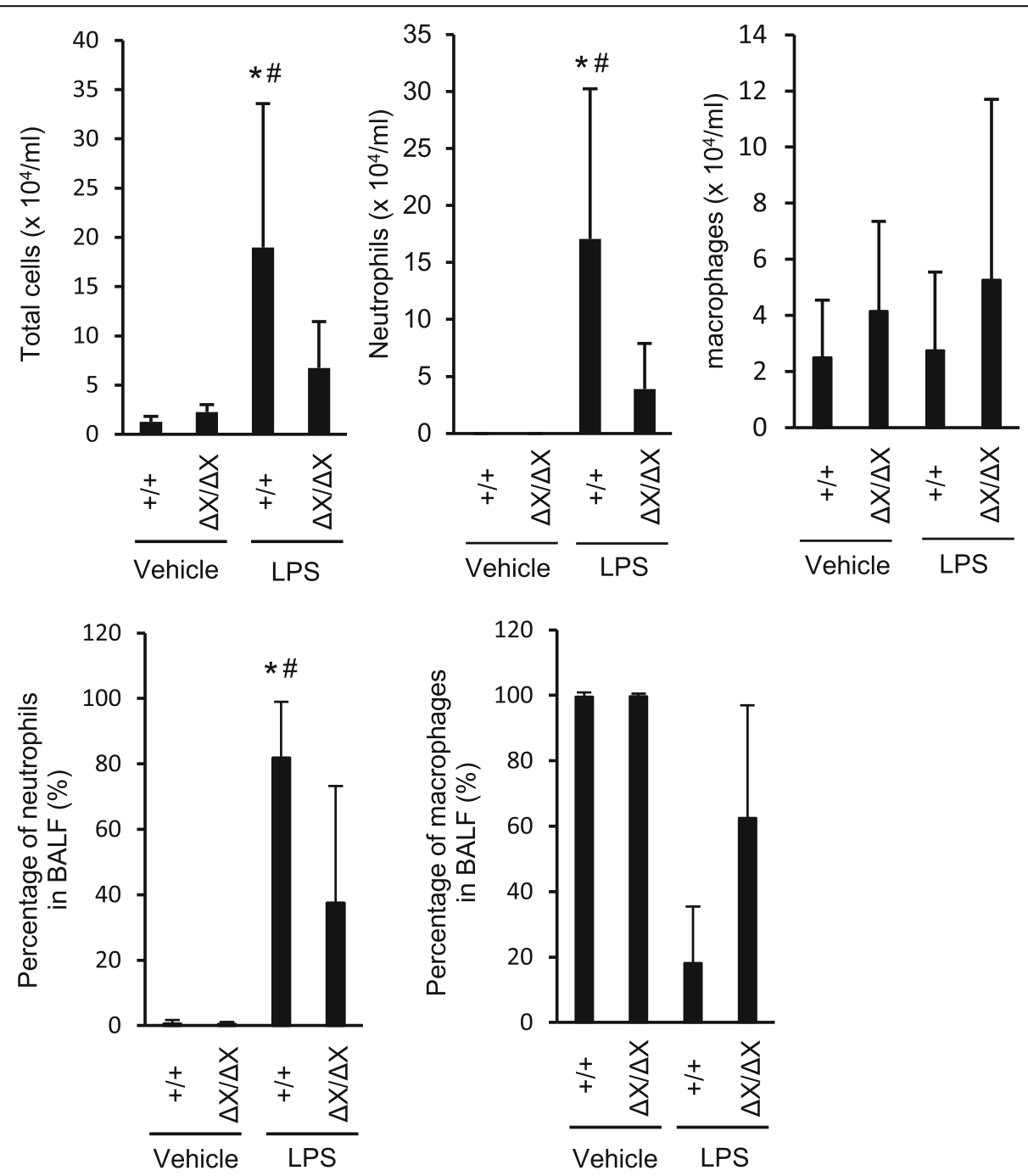

Fig. 2 Effects of the PLCE genotypes on neutrophilic inflammation in LPS-induced ALI. BALF was collected in $24 \mathrm{~h}$ after i.t. administration of LPS or vehicle, cytospinned, and stained with a Romanowski stain (Diff-Quik). Leukocytes and neutrophils were counted under a microscope. $n=9$ in the LPS-treated group, and $n=6$ in the vehicle-treated group. ${ }^{*}, p<0.05$ between control and LPS administration, \#, $p<0.05$ between PLCE genotypes 
inflammation accompanying the LPS-induced experimental ALI.

\section{Crucial role of PLC $\varepsilon$ in chemokine production in AECs accompanying the LPS-induced ALI}

We examined the mRNA levels of various proinflammatory cytokines in the whole lungs dissected from $P L C \varepsilon^{+/}$ ${ }^{+}$and $P L C \varepsilon^{\triangle X / \Delta X}$ mice in $24 \mathrm{~h}$ after the LPS administration by qRT-PCR. In the $P L C \varepsilon^{+/+}$lungs, the expression levels of various cytokines, such as Ccl2/MCP1, Ccl3/ MIP-1a, Ccl5/RANTES, Ccl9/MIP-1 g, Ccl11, Ccl12, Ccl22, Cxcl1/GRO-a, Cxcl2/MIP-2, Cxcl5/LIX/GCP-2, Cxcl9/MIG, Cxcl10/IP-10, Cxcl11/I-TAC, Cxcl13/BLC/ BCA-1,Cxcl15/Lungkine, IL-1 $\beta$, IL-6 and TNF- $\alpha$, showed marked elevation following the LPS administration (Fig. 3 and Additional file 1: Figure S2). Among them, the LPS-induced expression levels of $\mathrm{Ccl} 2, \mathrm{Ccl} 3$, Ccl5, Ccl11, Ccl12, Ccl22, Cxcl1, Cxcl2, Cxcl5, Cxcl9, Cxcl10, Cxcl11, Cxcl 13,Cxcl15, IL-1 $\beta$, IL-6 and TNF- $\alpha$ showed substantial reduction in $P L C \varepsilon^{\Delta X / \Delta X}$ mice $(p<$ 0.05, Fig. 3 and Additional file 1: Figure S2). Intriguingly, they contained Cxcl1, Cxcl2, Cxcl5 and Cxcl15, which function as the chemo-attractants for neutrophils [24]. Among them, we focused on $\mathrm{Cxcl} 5$ because it was known to be produced mainly by AECs and induce neutrophil trafficking in response to LPS exposure in the lung [24]. To clarify the nature of the cells expressing Cxcl5, we carried out immunohistochemical staining of the lung sections prepared from $P L C \varepsilon^{+/+}$and $P L C \varepsilon^{\Delta X / \Delta X}$ mice in $24 \mathrm{~h}$ after the LPS administration. The result showed that the LPS-induced increase in the Cxcl5 levels was observed mainly in the AECs, which was substantially attenuated in $P L C \varepsilon^{\Delta \mathrm{X} / \Delta \mathrm{X}}$ mice (Fig. 4a). The concentration of Cxcl5 in BALF also exhibited a great increase following the LPS administration in $P L C \varepsilon^{+/+}$ mice, which was almost completely suppressed in $P L C \varepsilon^{\Delta X / \Delta X}$ mice (Fig. 4b). These findings suggested an augmenting role of PLCE in the production of $\mathrm{Cxcl} 5$ in the exudative phase.

\section{Crucial role of PLC $\varepsilon$ in chemokine production in cultured AECs}

We next examined the role of PLCE in LPS-induced Cxcl5 expression by using primary cultures of AECs. The primary cultured AECs established from $P L C \varepsilon^{+/+}$ mice exhibited a marked increase in the Cxcl5 mRNA levels in $16 \mathrm{~h}$ after LPS stimulation, which was largely suppressed in those established from $P L C \varepsilon^{\Delta X / \Delta X}$ mice

Total Lung

\begin{tabular}{|c|c|c|c|c|c|}
\hline & $\begin{array}{c}\text { LPS } \\
(+/+/ \Delta \mathrm{X} / \Delta \mathrm{X})\end{array}$ & $P$ value & & $\begin{array}{c}\text { LPS } \\
(+/+/ \Delta \mathrm{X} / \Delta \mathrm{X})\end{array}$ & $P$ value \\
\hline Cc/2 & 5.61 & 0.001 & Cxcl1 & 2.52 & 3.4E-0.4 \\
\hline Cc/3 & 8.19 & 3.9E-06 & $\mathrm{Cxc} / 2$ & 2.20 & 2.7E-04 \\
\hline Ccl5 & 3.01 & 4.8E-04 & Cxc/5 & 4.39 & 3.6E-0.5 \\
\hline Cc/6 & 1.47 & 0.12 & Cxcl9 & 9.08 & $1.9 \mathrm{E}-05$ \\
\hline Ccl8 & 1.76 & $1.4 \mathrm{E}-04$ & Cxcl10 & 7.06 & $1.5 \mathrm{E}-05$ \\
\hline Cc/9 & 1.87 & 0.057 & Cxcl11 & 13.24 & 6.4E-05 \\
\hline Ccl11 & 3.36 & 0.0022 & Cxcl12 & 1.21 & 0.07 \\
\hline Ccl12 & 3.33 & $7.2 \mathrm{E}-05$ & Cxcl13 & 6.47 & $3.0 \mathrm{E}-04$ \\
\hline Cc/21 & 1.10 & 0.36 & Cxcl15 & 2.46 & $1.2 \mathrm{E}-05$ \\
\hline Ccl22 & 2.11 & $9.6 \mathrm{E}-04$ & Cxcl16 & 2.13 & $1.8 \mathrm{E}-04$ \\
\hline Ccl27 & 0.59 & 0.014 & $C \times 3 c / 1$ & 1.01 & 0.87 \\
\hline $\mathrm{Cc} / 28$ & 1.96 & 0.37 & C5a & 0.71 & 0.03 \\
\hline Chemerin & 1.98 & 0.014 & $I I-16$ & 2.38 & $5.6 \mathrm{E}-04$ \\
\hline$T N F-\alpha$ & 2.80 & 0.0057 & $I L-1 \beta$ & 5.78 & 1.4E-04 \\
\hline$I L-6$ & 4.27 & 1.4E-04 & & & \\
\hline
\end{tabular}

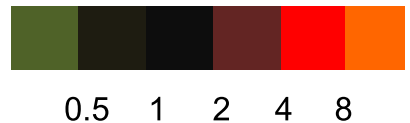

Fig. 3 Role of gene expression of chemokines after LPS stimulation in mice lungs. Lungs from mice with the indicated PLC $\varepsilon$ genotype was collected in $24 \mathrm{~h}$ after i.t. administration of LPS or vehicle. The RNA was pooled from each group ( $n=7$ in the LPS-treated group, $n=4$ in the vehicle-treated group) and subjected to qRT-PCR. Fold change was determined by dividing the relative mRNA level in $P L C \varepsilon^{+/+}$mice with that in $P L C \varepsilon^{\Delta \mathrm{X} / \Delta \mathrm{X}}$ mice. Values represent $p$-values between $P L C \varepsilon^{+/+}$and $P L C \varepsilon^{\Delta \mathrm{X} / \Delta \mathrm{X}}$ mice 


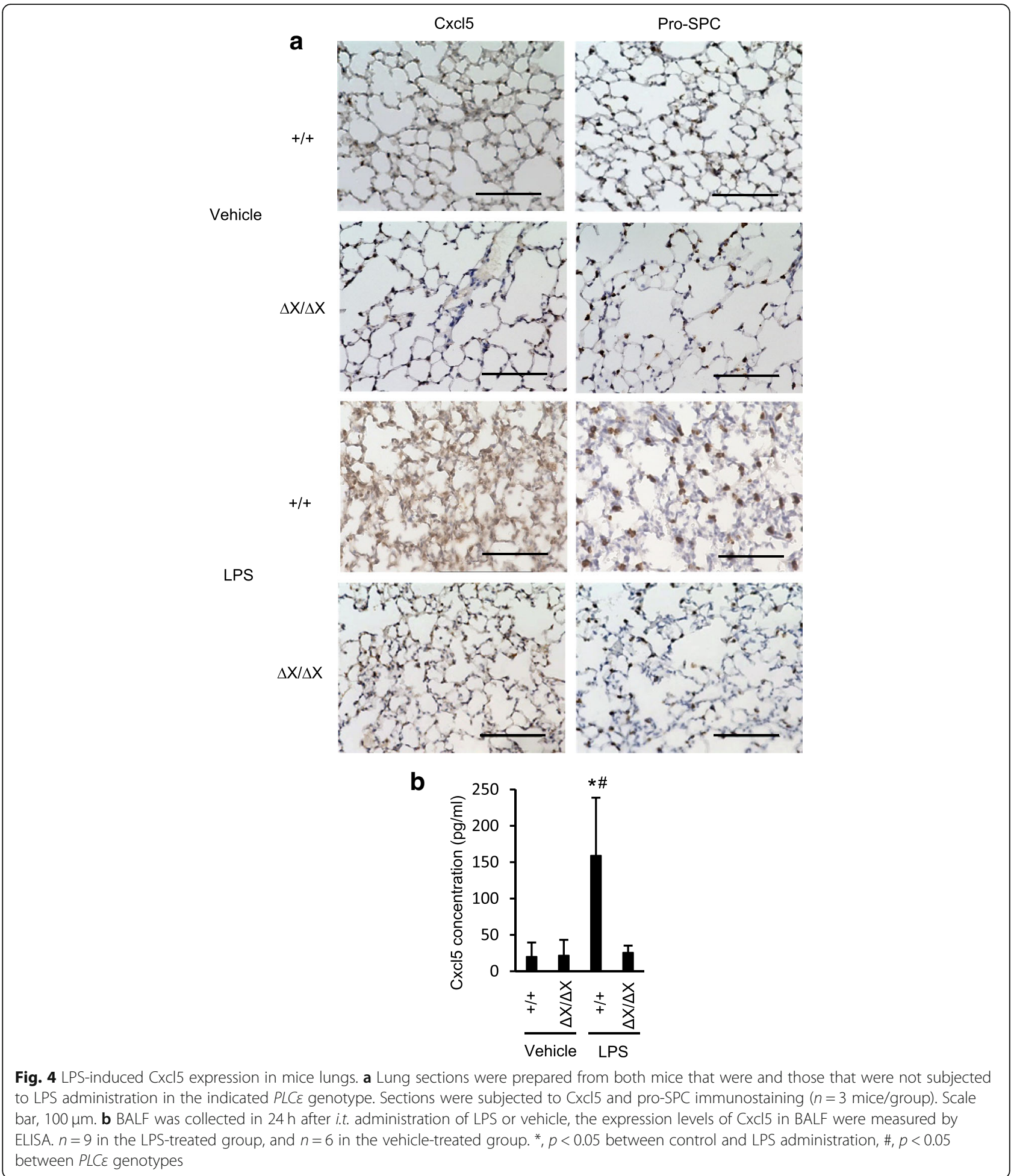

(Fig. 5a). Also, mmunofluorescence staining was used to confirm the LPS-induced $\mathrm{Cxcl} 5$ expression in $P L C \varepsilon^{+/+}$ AECs and its suppression in $P L C \varepsilon^{\Delta \mathrm{X} / \Delta \mathrm{X}}$ AECs (Fig. 5b). Thus, the PLCE-dependent augmentation of the LPS-induced Cxcl5 expression was recapitulated in vitro. Concurrently, the expression of Cxcl1, Cxcl2 and
Cxcl15, Ccl2, Ccl3, Ccl5, Ccl11, Ccl21 and Ccl27 was elevated in a manner similar to that of $\mathrm{Cxcl} 5$ (Additional file 1: Figure S3A, B). By contrast, the elevated expression of Cxcl10, Cxcl11, Ccl12, Ccl22, TNF- $\alpha$, IL-1 $\beta$ and IL-6 depending on the LPS stimulation and the $P L C \varepsilon^{+/+}$ background, occurred in the whole lung (Fig. 3 and 

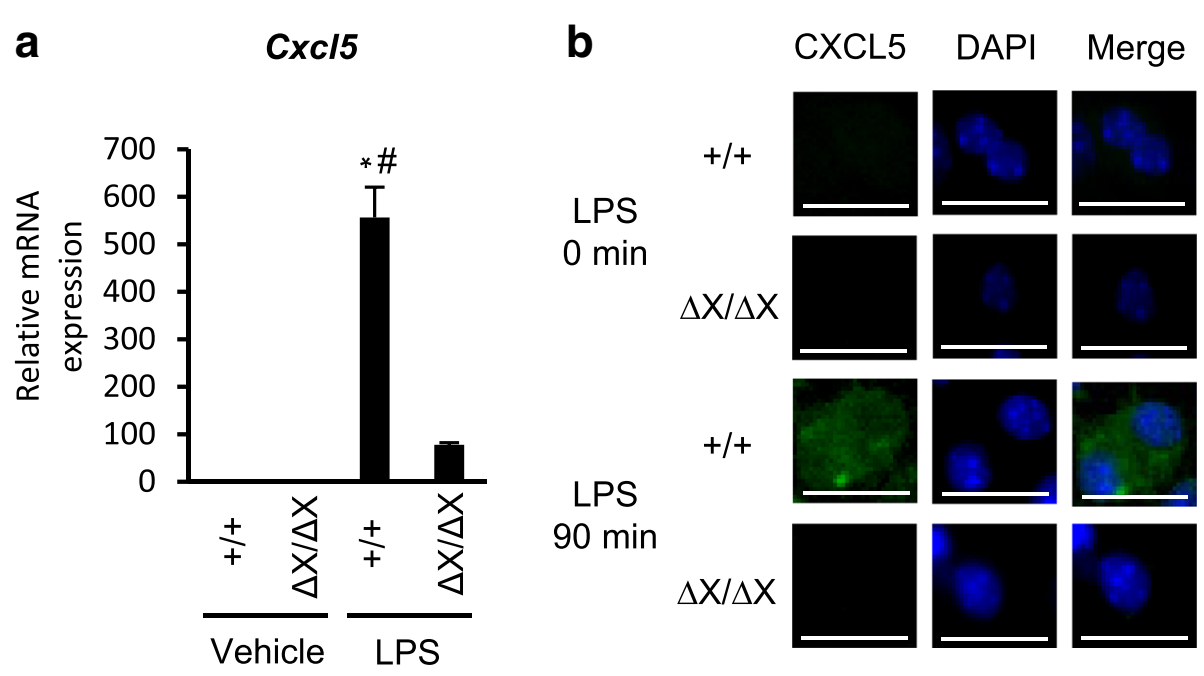

Fig. 5 Role of PLCE in LPS-induced chemokine production. a The RNA was collected from the primary cultures of $P L C \varepsilon^{+/+}$and $P L C \varepsilon^{\Delta X / \Delta X} A E C s$ in $16 \mathrm{~h}$ after the administration of LPS (500 $\mathrm{ng} / \mathrm{mL})$ or vehicle ( $n=8$ mice/group). The RNAs were pooled from each group and subjected to qRTPCR. ${ }^{*}, p<0.05$ between control and LPS administration, $\#, p<0.05$ between $P L C \varepsilon$ genotypes. b Primary cultured lung AECs from PLC $\varepsilon^{+/+}$and $P L C \varepsilon^{\triangle X / \Delta X}$ mice were prepared for CXCl5 (green) immunostaining after stimulation for $90 \mathrm{~min}$ by LPS ( $\left.500 \mathrm{ng} / \mathrm{ml}\right)(n=8$ mice/group). The Cxcl5 antibody $(15 \mu \mathrm{g} / \mathrm{ml})$ was used. Nuclei were counter-stained with 4',6-diamidino-2-phenylindole (DAPI) (blue). Scale bar, $25 \mu \mathrm{m}$

Additional file 1: Figure S2), was not observed in AECs. These differences might be accounted for by the contribution of cells different from AECs, such as infiltrated immune cells present in the lung.

\section{Crucial role of the PLCE-PKD-NF-KB signaling in LPS- induced $\mathrm{Cxcl} 5$ expression}

LPS is known to induce NF- $\mathrm{kB}$ activation through engagement of TLR4 via the canonical pathway, where the phosphorylation of IKB by IKK causes ubiquitination and subsequent proteasome degradation of $І$ І $B$, thereby allowing NF- $\mathrm{kB}$, a dimer of p65 RelA and p50, to undergo nuclear translocation and activate the expression of the target genes including those of various chemokines in an early time course [25]. We showed that, in human colon epithelial Caco2 cells, the activation of the canonical NF- $\mathrm{kB}$ pathway enhances the production and secretion of LPA, which activates the PLCE-PKD axis in an autocrine manner and leads to activation and cytoplasmic localization of RSK. The activated RSK, instead of IKK, phosphorylates IKB in the cytoplasm and induces its degradation, thereby activating NF- $\mathrm{KB}$ in a late time course [13]. To gain an insight into the molecular mechanisms of the PLC $\varepsilon^{\prime}$ s action, we examined the effects of the inhibitors of IKK and PKD on Cxcl5 expression in cultured $P L C \varepsilon^{+/+}$AECs in $16 \mathrm{~h}$ after the LPS stimulation, which corresponded to the late phase of NF- $\kappa \mathrm{B}$ activation. As a result, the LPS-induced elevation of the Cxcl5 mRNA level was almost completely abolished by the treatment with the IKK inhibitor while it was substantially suppressed by the treatment with the PKD inhibitor (Fig. 6a). Also, the nuclear translocation of the p65 subunit of NF-kB observed in $P L C \varepsilon^{+/+}$AECs was largely suppressed in $P L C \varepsilon^{\Delta X / \Delta X}$ AECs in $90 \mathrm{~min}$ after LPS stimulation, which also corresponded to the late phase of NF- $\mathrm{KB}$ activation (Fig. 6b). Likewise, the LPS-induced increase in the phosphorylation of PKD and IкB in $P L C \varepsilon^{+/+}$AECs was substantially suppressed in $P L C \varepsilon^{\Delta X / \Delta X}$ AECs (Fig. 6c). These results indicated that PLC $\varepsilon$ plays a crucial role in LPS-induced NF- $\mathrm{KB}$ activa-

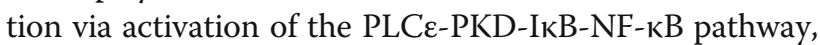
thereby augmenting the expression of the neutrophil-attracting chemokine Cxcl5.

\section{Discussion}

We demonstrated that PLCe plays a crucial role in the development of LPS-induced experimental ALI. By employing a similar experimental ALI model, Bijli et al. had reported a crucial role of PLCe in mediating endothelial cell inflammation and barrier disruption [26]. In the present study, we focused on the role of PLCE in AECs, which had been shown to act as the main source of pro-inflammatory cytokines and chemokines and thereby play a key role in the pathogenesis and resolution of ALI through regulation of neutrophil influx [27]. Also, AECs are known to play a pivotal role in innate immunity associated with the expression of TLR2 and TLR4 $[28,29]$. TLR4 is a major receptor of LPS, and the LPS/TLR4 signaling is relayed by activation of the MyD88-dependent and MyD88-independent pathways, inducing the mRNA expression of pro-inflammatory cytokines and type I interferon [30, 31].

Our results using the lung tissues and primary AEC cultures from $P L C \varepsilon^{+/+}$and $P L C \varepsilon^{\Delta X / \Delta X}$ mice suggested 
a
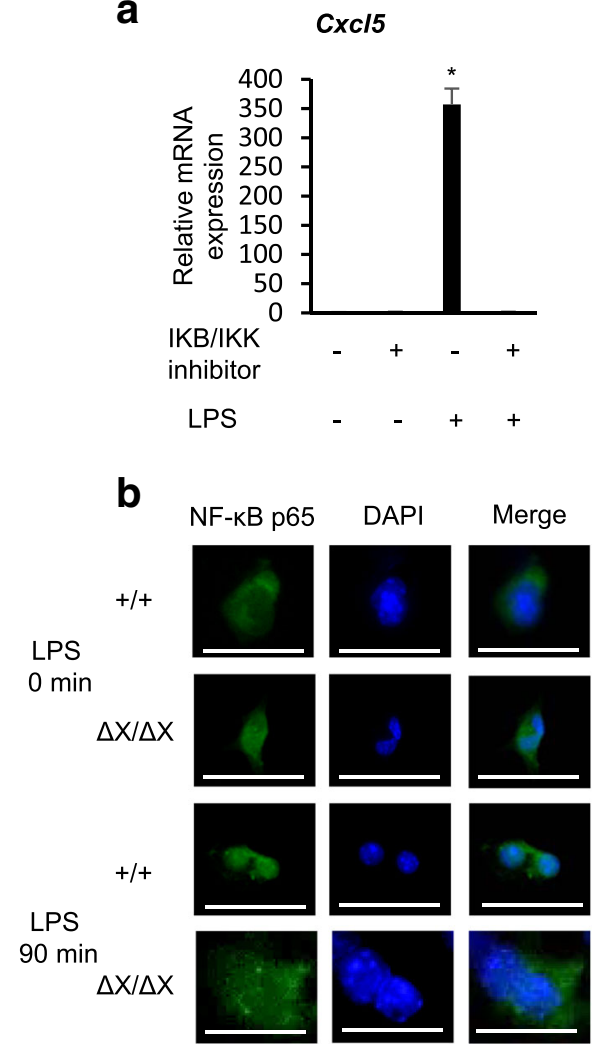

Cxc/5

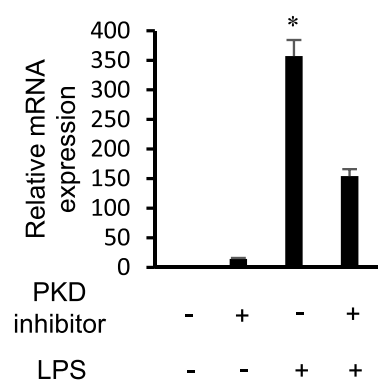

C

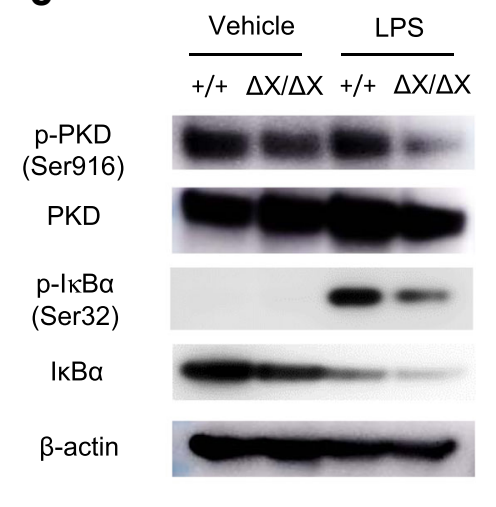

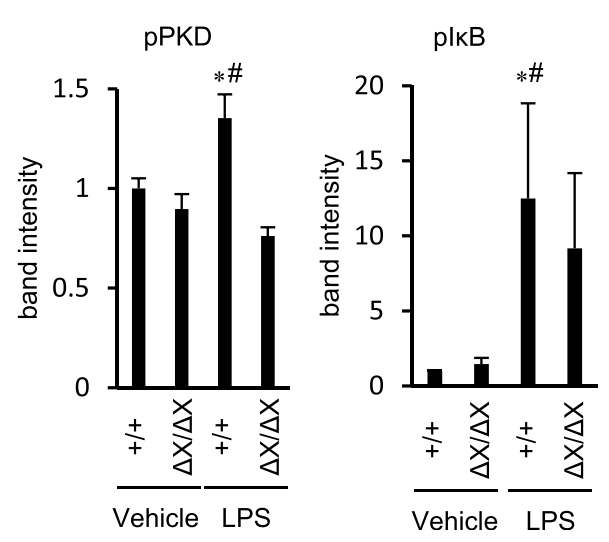

Fig. 6 Role of PLC $\varepsilon$ in LPS-induced NF-KB activation. a Primary-cultured AECs from PLC $\varepsilon^{+/+}$mice were treated with and without the IKB/IKK inhibitor $(20 \mu \mathrm{M})$ or the PKD inhibitor $(10 \mu \mathrm{g} / \mathrm{ml}) 0.5 \mathrm{~h}$ and $1 \mathrm{~h}$ before administration of LPS $(500 \mathrm{ng} / \mathrm{mL})$ or vehicle for $16 \mathrm{~h}$. Expression of the CXCl5 gene was analyzed by using qRT-PCR ( $n=7 /$ group). Values represent $p$-values between LPS-stimulated AECs and LPS-stimulated AECS with the IKB/IKK inhibitor or the PKD inhibitor. ${ }^{*}, p<0.05$. b Primary cultured AECs from $P L C \varepsilon^{+/+}$and $P L C \varepsilon^{\Delta X / \triangle X}$ mice were subjected to NF-KB $p 65$ (green) immunostaining after stimulation for 90 min with LPS (500 ng/ml) $(n=8$ mice/group). The anti-NF-KB p65 antibody (1:50) was used. Nuclei were counter-stained with DAPI (blue). Scale bar, $25 \mu \mathrm{m}$. c Proteins, prepared from the primary cultures of $P L C \varepsilon^{+/+}$and $P L C \varepsilon^{\Delta X / \Delta X}$ lung AECs in 1.5 $\mathrm{h}$ after treatment with LPS $(500 \mathrm{ng} / \mathrm{ml})$ or vehicle ( $n=8$ mice/group) were subjected to Western blotting with the indicated antibodies (upper panel). The intensity of each phospho-protein band was divided by the intensity of each total protein band. The average intensities obtained from at least three independent experiments were shown as the mean \pm S. D with $p$-values (lower panels). ${ }^{*}, p<0.05$ between control and LPS administration, \#, $p<0.05$ between $P L C \varepsilon$ genotypes

that PLCe might facilitate LPS-induced neutrophilic inflammation through augmentation of the production of the CXC family of chemokines, in particular Cxcl5, from AECs. Among the LPS-induced proinflammatory cytokines, we focused our study on chemokines that attract and activate neutrophils, in particular the CXC chemokines possessing the CXC cysteine motif [32]. The CXC chemokines are divided into two subgroups depending on the presence or absence of the Glu-Leu-Arg (ELR) motif. The CXC chemokines with the ELR motif, 
exemplified by Cxcl1, Cxcl2, Cxcl5 and Cxcl15, induce neutrophil chemotaxis, and $\mathrm{Cxcl} 1, \mathrm{Cxcl} 2, \mathrm{Cxcl} 5$ and Cxcl15 had been reported to function as important neutrophil chemo-attractants associated with lung inflammation in mice [32, 33]. Among them, we focused on Cxcl 5 because it is produced mainly by AECs and because it is known to induce neutrophil trafficking in response to LPS exposure in the lung [24]. A key role of Cxcl5 had been shown in lung inflammation such as that occurred in the mouse model of pneumococcal pneumonia [34, 35]. Moreover, it had been reported that neutralization of $\mathrm{Cxcl} 5$ in the lung suppressed neutrophilic inflammation in a mouse model of LPS-induced ALI [6]. Furthermore, it had been observed that the levels of Cxcl5 in BALF of patients with ARDS were high [36-38]. These findings strongly supported our notion that the PLCe-mediated production of $\mathrm{Cxcl} 5$ from AECs might be causal to the pathogenesis of the LPS-induced ALI.

It is known that the expression of CXC chemokines including Cxcl5 is regulated by NF- $\mathrm{BB}[39,40]$. The expression of Cxcl5 accompanying pneumococcal pneumonia had been shown to be induced by NF- $\mathrm{kB}$ activation [33]. Here we showed that PLCe augments LPS-induced Cxcl5 expression through NF- $\mathrm{kB}$ activation via the PLCe-PKD-IкB pathway in AECs. This pathway had been demonstrated to be responsible for the late phase of NF- $\mathrm{KB}$ activation upon stimulation of cultured human colon epithelial cells by TNF- $\alpha$, while the early phase was mediated by the canonical pathway using IKK for $I_{\kappa} B$ phosphorylation and linked to the late phase by LPA [13]. In AECs, it is likely that the link between the early and late phases is provided by S1P, which was known to induce PLCe activation [9] and cooperate with the TLR4 signaling to enhance cytokine production in human gingival epithelial cells [41] through engagement of its GPCR receptors. The importance of the PLCE-PKD-IкB pathway in mediating NF- $k B$ activation downstream of TLRs had been suggested by the observations that PKD was essential for the MyD88-dependent TLR signaling [42] and that PKD was involved in chemokine release induced by TLR2, TLR4 and TLR5 [43]. In our present study, the evidence for the critical role of the PLCE-PKD-IKB pathway came from the observation that the LPS-induced Cxcl5 expression was suppressed by the inhibitors of IKK and PKD and that the LPS-induced nuclear translocation of $\mathrm{NF}-\mathrm{\kappa B}$ and phosphorylation of PKD and I $\mathrm{KB}$ were suppressed in $P L C \varepsilon^{\Delta X / \Delta X}$ AECs. The IKK inhibitor was expected to suppress both the early and late phases of NF- $\kappa B$ activation while the PKD inhibitor was expected to suppress only the late phase. Our present observation that the PKD inhibitors resulted in substantial inhibition of LPS-induced Cxcl5 expression suggested that the
PLCe-PKD-I $\mathrm{KB}$ pathway played a major role in the late phase NF-kB activation.

\section{Conclusions}

The results, taken together, indicated that PLCe plays a crucial role in neutrophilic inflammation accompanying LPS-induced experimental ALI through augmentation of Cxcl5 production in AECs via activation of the

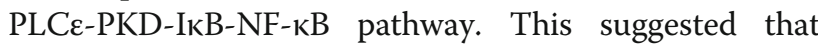
PLC $\varepsilon$ might become a promising candidate target for the treatment of ARDS, which was also supported by the fact that $P L C \varepsilon^{\Delta X / \Delta X}$ mice were born and grew normally except for the development of mild abnormality in the heart valves [44] predicting the low toxicity of PLCE-specific inhibitors.

\section{Additional files}

Additional file 1: Figure S1. Primary cultured AECs with

immunohistochemical staining. Figure S2. Effect of PLC $\varepsilon$ on expression

of chemokines and cytokines in LPS-induced ALI mice (related to Fig. 3). Figure S3A. Comparison of the expression levels of LPS-induced chemokine and cytokine(related to Fig. 5). Figure S3B. Role of PLC $\varepsilon$ in LPSinduced chemokine and cytokine production (related to Fig. 5). (PPTX 1631 kb)

Additional file 2: Table S1. List of primer sequences. (DOCX $21 \mathrm{~kb}$ )

\section{Abbreviations}

AEC: Alveolar epithelial cell; ALI: Acute lung injury; ARDS: Acute respiratory distress syndrome; BALF: Bronchoalveolar lavage fluid; CCL: Chemokine C-C motif ligand; CXCL: Chemokine C-X-C motif ligand; DAG: Diacylglycerol; ELR: Glu-Leu-Arg; GPCR: G protein-coupled receptor; H\&E: Hematoxylin and eosin; IKK: Inhibitor KB kinase; IKB: Inhibitor KB; LPA: Lysophosphatidic acid; LPS: Lipopolysaccharide; MyD88: Myeloid differentiation primary response gene 88; NF-kB: Nuclear factor-kB; PBS: Phosphate- buffered saline;

PKD: Protein kinase D; PLC: Phospholipase C; pro-SPC: Prosurfactant protein C; qRT-PCR: Quantitative reverse transcription-polymerase chain reaction;

S1P: Sphingosine-1-phosphate; SD: Standard deviation; TLR: Toll-like receptor

\section{Acknowledgments}

We thank Dr. Hironori Edamatsu of the Division of Molecular Biology and all the members of the Division of Respiratory Medicine, Kobe University Graduate School of Medicine, for their helpful discussions.

Funding

This work was supported by JSPS KAKENHI 26461188 to Kazuyuki Kobayashi.

Availability of data and materials

All data generated or analyzed during this study are included in this article.

\section{Authors' contributions}

KU and TN participated in study design. KU, TN, KK and TK wrote the manuscript. All authors analyzed the data and KK conceived the study. MY conducted the statistical analysis. TK and YN assisted with technical advice. All authors read and approved the final manuscript.

\section{Ethics approval}

All the animal experiments were approved by the Institutional Animal Care and Use Committee (Permit Numbers: P130612, P130612-R1, and P130401) and carried out in accordance with the Kobe University Animal Experimentation Regulations. 


\section{Competing interests}

The authors declare that they have no competing interests.

\section{Publisher's Note}

Springer Nature remains neutral with regard to jurisdictional claims in published maps and institutional affiliations.

\section{Author details}

'Division of Respiratory Medicine, Department of Internal Medicine, Kobe University Graduate School of Medicine, 7-5-1 Kusunoki-cho, Chuo-ku, Kobe 650-0017, Japan. ²Division of Molecular Biology, Department of Biochemistry and Molecular and Biology, Kobe University Graduate School of Medicine, 7-5-1 Kusunoki-cho, Chuo-ku, Kobe 650-0017, Japan. ${ }^{3}$ Kobe University Incubation Center, 1-5-6 Miyakojima Minami-cho, Chuo-ku, Kobe 650-0047, Japan

Received: 7 October 2018 Accepted: 2 January 2019

Published online: 11 January 2019

\section{References}

1. Thompson BT, Chambers RC, Liu KD. Acute respiratory distress syndrome. $\mathrm{N}$ Engl J Med. 2017;377:562-72.

2. Chiumello D, Coppola S, Froio S, Gotti M. What's next after ARDS: long-term outcomes. Respir Care. 2016;61:689-99.

3. Marshall $R$, Bellingan $G$, Laurent $G$. The acute respiratory distress syndrome: fibrosis in the fast lane. Thorax. 1998:53:815-7.

4. Ware LB, Matthay MA. The acute respiratory distress syndrome. N Engl J Med. 2000;342:1334-49.

5. Gropper MA, Kronish JW. The epithelium in acute lung injury/acute respiratory distress syndrome. Curr Opin Crit Care. 2008;14:11-5.

6. Jeyaseelan S, Manzer R, Young SK, Yamamoto M, Akira S, Mason RJ, et al. Induction of CXCL5 during inflammation in the rodent lung involves activation of alveolar epithelium. Am J Respir Cell Mol Biol. 2005;32:531-9.

7. Smrcka AV, Brown JH, Holz GG. Role of phospholipase C $\varepsilon$ in physiological phosphoinositide signaling networks. Cell Signal. 2012;24:1333-43.

8. Suh PG, Park JI, Manzoli L, Cocco L, Peak JC, et al. Multiple roles of phosphoinositide-specific phospholipase C isozymes. BMB Rep. 2008;41: 415-34.

9. Kelley GG, Reks SE, Smrcka AV. Hormonal regulation of phospholipase Cepsilon through distinct and overlapping pathways involving G12 and Ras family G-proteins. Biochem J. 2004;378:129-39.

10. Ikuta S, Edamatsu H, Li M, Hu L, Kataoka T. Crucial role of phospholipase C $\varepsilon$ in skin inflammation induced by tumor-promoting phorbol ester. Cancer Res. 2008:68:64-72.

11. Hu L, Edamatsu H, Takenaka N, Ikuta S, Kataoka T. Crucial role of phospholipase $C \varepsilon$ in induction of local skin inflammatory reactions in the elicitation stage of allergic contact hypersensitivity. J Immunol. 2010;184: 993-1002.

12. Nagano T, Edamatsu H, Kobayashi K, Takenaka N, Yamamoto M, Sasaki N, et al. Phospholipase $C \varepsilon$, an effector of Ras and rap small GTPases, is required for airway inflammatory response in a mouse model of bronchial asthma. PLoS One. 2014;9:e108373.

13. Wakita M, Edamatsu H, Li M, Emi A, Kitazawa S, Kataoka T. Phospholipase C activates nuclear factor-KB signaling by causing cytoplasmic localization of ribosomal $\mathrm{S} 6$ kinase and facilitating its phosphorylation of inhibitor $\mathrm{kB}$ in colon epithelial cells. J Biol Chem. 2016;291:12586-600.

14. Takenaka N, Edamatsu $H$, Suzuki N, Saito H, Inoue $Y$, et al. Overexpression of phospholipase $C \varepsilon$ in keratinocytes upregulates cytokine expression and causes dermatitis with acanthosis and T-cell infiltration. Eur J Immunol. 2002;41:202-13

15. Bai $Y$, Edamatsu H, Maeda S, Saito H, Suzuki N, Satoh T, et al. Crucial role of phospholipase $C \varepsilon$ in chemical carcinogen-induced skin tumor development. Cancer Res. 2004;64:8808-10

16. Li M, Edamatsu H, Kitazawa R, Kitazawa S, Kataoka T. Phospholipase Cepsilon promotes intestinal tumorigenesis of $\mathrm{Apc}(\mathrm{Min} /+)$ mice through augmentation of inflammation and angiogenesis. Carcinogenesis. 2009:30: 1424-32.

17. Corti M, Brody AR, Harrison JH. Isolation and primary culture of murine alveolar type II cells. Am J Respir Cell Mol Biol. 1996;14:309-15.
18. Warshamana GS, Corti M, Brody AR. TNF-alpha, PDGF, and TGF- $\beta$ (1) expression by primary mouse bronchiolar-alveolar epithelial and mesenchymal cells: TNF-a induces TGF- $\beta$ (1). Exp Mol Pathol. 2001;71:13-33.

19. Kim SC, Kellett T, Wang S, Nishi M, Nagre N, Zhou B, et al. TRIM72 is required for effective repair of alveolar epithelial cell wounding. Am J Physiol Lung Cell Mol Physiol. 2014;307:L449-59.

20. Walker JE Jr, Odden AR, Jeyaseelan S, Zhang P, Bagby GJ, Nelson S, et al. Ethanol exposure impairs LPS-induced pulmonary LIX expression: alveolar epithelial cell dysfunction as a consequence of acute intoxication. Alcohol Clin Exp Res. 2009;33:357-65.

21. Hatakeyama Y, Kobayashi K, Nagano T, Tamura D, Yamamoto M, Tachihara $M$, et al. Synergistic effects of pemetrexed and amrubicin in non-small cell lung cancer cell lines: potential for combination therapy. Cancer Lett. 2014; 343:74-9.

22. Harada Y, Edamatsu H, Kataoka T. PLC $\varepsilon$ cooperates with the NF-KB pathway to augment TNFa-stimulated CCL2/MCP1 expression in human keratinocyte. Biochem Biophys Res Commun. 2011:414:106-11.

23. Matute-Bello G, Downey G, Moore BB, Groshong SD, Matthay MA, Slutsky AS, Acute Lung Injury in Animals Study Group, et al. An official American Thoracic Society workshop report: features and measurements of experimental acute lung injury in animals. Am J Respir Cell Mol Biol. 2011; 44:725-38.

24. Bhatia $\mathrm{M}$, Zemans $\mathrm{RL}$, Jeyaseelan $\mathrm{S}$. Role of chemokines in the pathogenesis of acute lung injury. Am J Respir Cell Mol Biol. 2012;46:566-72.

25. Natoli G, Chiocca S. Nuclear ubiquitin ligases, NF-kappaB degradation, and the control of inflammation. Sci Signal. 2008;1:pe1.

26. Bijli KM, Fazal F, Slavin SA, Leonard A, Grose V, Alexander WB, et al. Phospholipase $C-\varepsilon$ signaling mediates endothelial cell inflammation and barrier disruption in acute lung injury. Am J Physiol Lung Cell Mol Physiol. 2016:311:L517-24.

27. Manicone AM. Role of the pulmonary epithelium and inflammatory signals in acute lung injury. Expert Rev Clin Immunol. 2009:5:63-75.

28. Thorley A, Ford P, Giembycz M, Goldstraw P, Young A, Tetley T. Differential regulation of cytokine release and leukocyte migration by lipopolysaccharide-stimulated primary human lung alveolar type II epithelial cells and macrophages. J Immunol. 2007;178:463-73.

29. Armstrong L, Medford A, Uppington KM, Robertson J, Witherden IR, Tetley TD, et al. Expression of functional toll-like receptor-2 and -4 on alveolar epithelial cells. Am J Respir Cell Mol Biol. 2004;31:241-5.

30. Lu YC, Yeh WC, Ohashi PS. LPS/TLR4 signal transduction pathway. Cytokine. 2008:42:145-51

31. Sender V, Stamme C. Lung cell-specific modulation of LPS-induced TLR4 receptor and adaptor localization. Commun Integr Biol. 2014;7:e29053-1-9.

32. Puneet $P$, Moochhala $S$, Bhatia $M$. Chemokines in acute respiratory distress syndrome. Am J Physiol Lung Cell Mol Physiol. 2005;288:L3-15.

33. Yamamoto K, Ahyi AN, Pepper-Cunningham Z, Ferrari JD, Wilson AA, Jones $M R$, et al. Roles of lung epithelium in neutrophil recruitment during pneumococcal pneumonia. Am J Respir Cell Mol Biol. 2013;50:253-62.

34. Mei J, Liu Y, Dai N, Favara M, Greene $T$, Jeyaseelan $S$, et al. CXCL5 regulates chemokine scavenging and pulmonary host defense to bacterial infection. Immunity. 2010:33:106-17.

35. Liu Y, Mei J, Gonzales L, Yang G, Dai N, Wang P, et al. IL-17A and TNF-a exert synergistic effects on expression of CXCL5 by alveolar type II cells in vivo and in vitro. J Immunol. 2011;186:3197-205

36. Goodman RB, Strieter RM, Martin DP, Steinberg KP, Millberg JA, Maunder RJ, et al. Inflammatory cytokines in patients with persistence of the acute respiratory distress syndrome. Am J Respir Crit Care Med. 1996;154:602-11.

37. Wiedermann FJ, Mayr AJ, Kaneider NC, Fuchs D, Mutz NJ, Schobersberger W. Alveolar granulocyte colony-stimulating factor and alpha-chemokines in relation to serum levels, pulmonary neutrophilia, and severity of lung injury in ARDS. Chest. 2004;125:212-9.

38. Keane MP, Donnelly SC, Belperio JA, Goodman RB, Dy M, Burdrick MD, et al. Imbalance in the expression of CXC chemokines correlates with bronchoalveolar lavage fluid angiogenic activity and procollagen levels in acute respiratory distress syndrome. J Immunol. 2002;169:6515-21.

39. Sun H, Chung WC, Ryu SH, Ju Z, Tran HT, Kim E, et al. Cyclic AMP-responsive element binding protein- and nuclear factor-kappaB-regulated CXC chemokine gene expression in lung carcinogenesis. Cancer Prev Res. 2008;1: $316-28$

40. Kim JM, Oh YK, Kim YJ, Oh HB, Cho YJ. Polarized secretion of CXC chemokines by human intestinal epithelial cells in response to Bacteroides 
fragilis enterotoxin: NF-KB plays a major role in the regulation of IL-8 expression. Clin Exp Immunol. 2001;123:421-7.

41. Eskan MA, Rose BG, Benakanakere MR, Zeng Q, Fujioka D, Martin MH, et al. TLR4 and S1P receptors cooperate to enhance inflammatory cytokine production in human gingival epithelial cells. Eur J Immunol. 2008;38:1138-47.

42. Park JE, Kim Yl, Yi AK. Protein kinase D1 is essential for MyD88-dependent TLRsignaling pathway. J Immunol. 2009;182:6316-27.

43. Steiner TS, Ivison SM, Yao Y, Kifayet A. Protein kinase D1 and D2 are involved in chemokine release induced by toll-like receptors 2,4 , and 5 . Cell Immunol. 2010;264:135-42.

44. Tadano M, Edamatsu H, Minamisawa S, Yokoyama U, Ishikawa Y, Suzuki N, et al. Congenital semilunar valvulogenesis defect in mice deficient in phospholipase Cr. Mol Cell Biol. 2005;25:2191-9.

Ready to submit your research? Choose BMC and benefit from:

- fast, convenient online submission

- thorough peer review by experienced researchers in your field

- rapid publication on acceptance

- support for research data, including large and complex data types

- gold Open Access which fosters wider collaboration and increased citations

- maximum visibility for your research: over $100 \mathrm{M}$ website views per year

At $\mathrm{BMC}$, research is always in progress.

Learn more biomedcentral.com/submissions 